\title{
Professional resilience skill training (pro-rest): an evidence-based and feasible educational module
}

\author{
Roslan, N.S. ${ }^{1}$, Yusoff, M.S.B. ${ }^{1}$, Morgan, K. $^{2}$, Razak, A.A. ${ }^{1}$, Shauki, N.I.A. ${ }^{3}$ \\ ${ }^{1}$ Universiti Sains Malaysia, ${ }^{2}$ RCSI-Perdana University, ${ }^{3}$ Ministry of Health Malaysia
}

\begin{abstract}
Physicians burnout has been regarded as a public health crisis. Medical interns are at a higher risk to develop burnout and other mental health problems. As organizational newcomers, interns face a challenging transition from undergraduate training to patient care responsibilities. Interns have a lower job autonomy that places them in a high strain category. While there is continuous training to improve their knowledge and skills, resilience training is not a common practice across centers. Most of the available interventions in the literature require continuous participation and are not practical within the internship training context. However, an effective and feasible resilience intervention is critical alongside the organizational improvements that may take a longer implementation time. Hence, we set out to develop a Professional Resilience Skill Training (Pro-ReST) module to enhance professional resilience among medical interns. In the development phase, we begin by examining the prevalence of mental health problems and the conceptualization of resilience during the internship through in-depth interviews (IDI) with various stakeholders in ten training hospitals across Malaysia. We then combined the findings with the DEAL model (Detection, and Evaluation of stressor, Action and Learning) to develop an educational module. The module development was guided by the cognitive load theories to ensure that it can be delivered within four hours. We then conducted a validation study with the expert panels and graduated medical students. In the evaluation phase, we conducted a 10-week parallel single-blinded randomized controlled trial (RCT) in two training hospitals and diary studies to explore the role of the module in interns' daily encounters with adversities. $73.3 \%$ of interns had burnout syndrome. Interns who utilized maladaptive coping were 1.71 to 4.09 times more likely to develop burnout. Through the IDI, we found that resilience development during the internship is not only driven by tenacity, but also coping skills, support, teamwork, and reflection. As support is an important component to support newcomers' resilience, the module expanded the original DEAL model to WEDEAL (Detection, and Evaluation of stressor, Action, Learning and Helping Others). The new component enables interns to also support their colleagues' resilience development using a similar framework. Several measures were used to maintain an appropriate cognitive load during the module delivery (e.g. hands-on group activities). The module was found to have an excellent Content and Face Validity Index. In the evaluation study, the RCT revealed significantly higher resilience and planning coping scores, and lower anxiety, stress symptoms, and use of maladaptive coping scores in the intervention cohort. The diary study revealed that many participants were more aware of their coping mechanisms and able to practice effective coping strategies. However, the participants acknowledged that coping skills were not able to solve some superior-related stressors. The Pro-ReST module is an effective individual-targeted intervention to increase resilience among medical interns. It requires minimal training and can be delivered within four hours, making it a potential workplace training module in all hospitals during the internship training.
\end{abstract}

Keywords: Burnout, medical interns 